\title{
A Mobile Cloud based Approach for Secure Medical Data Management
}

\author{
Annapurna Patil, PhD \\ Dept. of CSE \\ M S Ramaiah Institute of \\ Technology \\ Bangalore, India
}

\author{
Ashwini D V \\ Dept. of CSE \\ M S Ramaiah Institute of \\ Technology \\ Bangalore, India
}

\author{
Rashmi Ranjana T P \\ Dept. of CSE \\ M S Ramaiah Institute of \\ Technology \\ Bangalore, India
}

\author{
Tulasi Srinivas \\ Dept. of CSE \\ M S Ramaiah Institute of \\ Technology \\ Bangalore, India
}

\begin{abstract}
Mobile cloud Computing has become part of cloud computing that also deals with various issues of cloud services when is accessed by portable devices in wireless environment. The Healthcare data is relatively sensitive compared to other data which need at most supervision. When Cloud services are accessed by the mobile devices additional challenge like security arises. Providing the Security and privacy for the medical data is an interesting topic to deal with. In this paper the survey is done about the various challenges faced in Mobile Cloud Computing and the existing scenarios.
\end{abstract}

\section{General Terms}

Cloud Computing, Healthcare Sector, Privacy.

\section{Keywords}

Mobile Cloud, Medical Data Management, Data Security.

\section{INTRODUCTION}

The world is driven by the digital technology. Smart phones have transformed our daily lives and our communication ways. The Medical sector which has enormous amount of data needs to be digitized in the form of electronic health records, hospital patient administrative system, laboratory systems etc.

A healthcare information system (HIS) is an important support tools in the management of health care services. It refers to any system that captures, stores, manages information related to the health of individuals or the activities of organizations that work within the health sector [1]. It has four key functions: (1) data generation (2) compilation (3) analysis and synthesis (4) communication and use. The HIS collects data from the health sector and other relevant sectors, analyses the data and ensures their overall quality, relevance and timeliness, and converts data into information for health-related decision-making [2].

The ability to exchange health information electronically helps in providing higher quality and safer care for patients in many ways like (1) Providing accurate, up-to-date, and complete information about patients at the point of care, (2) Enabling quick access to patient records for more coordinated, efficient care, (3) Helping providers more effectively diagnose patients, reduce medical errors, and provide safer care, (4) Improving patient and provider interaction and communication, as well as health care convenience, (5) Enabling safer, more reliable prescribing etc.

In today's world everyone looks for instantaneous and ubiquitous access to information. Anytime and anywhere access to information has become a de facto requirement. Cloud computing is such a paradigm which provides services on-demand to the users. The cloud can act as the foundation for connected health to support a range of complex, disparate, and mission-critical applications [5]. Cloud helps the hospitals and healthcare providers to use the applications, hardware, and services on a pay per use model. This allows them to avoid heavy investments on buying and deploying technology.

Mobile devices have many constraints because of their smaller sizes, lower weights, longer battery life and other features. Hence there is inflexibility in hardware and software development for these devices. Cloud computing can be used to permit the mobile devices to avoid these constraints by making the resource intensive tasks and complex functions to be performed on desktop systems and having the end results sent to the device [5]. Mobile Cloud Computing (MCC) is the combination of cloud computing, mobile computing and wireless networks to bring rich computational resources to mobile users, network operators, as well as cloud computing providers [4]. Thus MCC is a very efficient and effective way to develop robust applications in the healthcare sector. The users can share resources and applications without high investment on hardware and software resources. The users can easily run the applications from the mobile without the need of extra hardware to run the applications as the operations are run within the cloud.

The major challenge in mobile cloud computing is privacy and security. The Data maintained in a cloud may contain personal, private or confidential information such as healthcare related information that requires the proper safeguards to prevent disclosure, compromise or misuse. Globally, concerns related to data jurisdiction, security, privacy and compliance are impacting adoption by healthcare organizations ask that authors follow some simple guidelines. In essence, we ask you to make your paper look exactly like this document. The easiest way to do this is simply to download the template, and replace the content with your own material.

\section{MOTIVATION}

There are few open issues in the field of health care such as: (1) Maintenance of large patient data available which also include images from various devices like MRI scanner XR machines etc. (2) Inadequate trained workforce for handling electronic data. (3) Data sharing is usually un-common and lack of privacy with patients identification. All these leads to increase in the cost to avail good health care services are the motivations for this proposed framework.

The proposed framework solves the problem of managing patient data globally which is generated via different means, providing a feature for storage and retrieval of data securely on a Cloud DB. 
The paper is further organized as follows. In Section III, we present the related work of some of healthcare systems in cloud computing. In Section IV, we describe the system architecture of the proposed framework, while conclusions and final remarks are presented in last section.

\section{RESEARCH BACKGROUND}

This section provides review of research work conducted in the field of cloud computing for storing and sharing large data files involved in medical imaging. Current challenges faced in this area are also mentioned below.

\subsection{Cloud in Healthcare}

In the traditional setting, large scale storage is not provided while in cloud computing significant benefits are available for large scale storage of patient's data. Moreover the data should be shared across various parts of geography, helping the doctors to speed up the treatments. Cloud caters for all these requirements providing the health care organizations to provide better service to patients, also to improve the quality of treatment.

Increasing changes on the norms and acts applied, increased demand, and adoption of electronic medical record systems is forcing the medical data to be more secure and safe. There is increasing demand for Accessibility and availability of the data. When the healthcare sector planned to move to cloud, it should carefully move the applications, deciding the cloud service models that best address the business requirements.

Looking at the various models of cloud, SaaS, with its payper-use business model will be the most attractive economic option, especially for small physician practices, since the need for full-time IT personnel is eliminated along with capital expenses associated with system hardware, operating systems and software. PaaS is a viable option for larger healthcare institutions that have the resources to develop their own cloud based solutions. For healthcare institutions seeking a more scalable infrastructure, IaaS offers a cost-effective turn-key solution that provides scalability with security, flexibility, defined service level agreements, built-in backup and data protection.

\subsection{Mobile Cloud Computing in \\ Healthcare}

There is a huge technology development in the field of mobile devices. There is also a enormous demand for the storage of data produced by these hand held devices, due to the constraints like small size, lower weight, less battery life and other features. Due to which mobile devices are entering into cloud for storing there data.

Cloud computing allows these mobile devices capable of performing resource intensive tasks and complex functions, by this mobile devices can develop efficient robust applications in health care sector. The barrier for invention of newer technology in healthcare sector using mobile devices are reduced by using cloud computing. The users can access the patient data from any part and anywhere of the world.

In the future, cloud solutions will enable healthcare providers to place remote mobile diagnostic devices in patients' homes, link these to cloud platforms, and monitor them continually with applications, including cloud-based predictive analytics. In combination, these elements will enable patients with chronic conditions to stay in their own homes, while giving providers the ability to track treatments and take action whenever necessary-including anticipating and intervening to address health problems before they arise [6].
3.3 Healthcare Systems - Existing Scenario

The existing solutions about cloud in health environment involve different aspects. The categorization involves:

\subsubsection{Medical Images archive solutions in the Cloud}

There are various technologies to store the electronically generated medical image. Using Microsoft Azure a prototype was proposed where DICOM (Digital Imaging and Communications in Medicine) servers were used to handle storage, querying and retrieving user requested images. The images were stored in SQL Azure database, where the web UI was also provided. Sharing exchanging and storing of images between different hospitals issues were resolved by one of the proposal called MIFAS (Medical Image File Accessing System).

With this technology advancement security plays a vital role in accessing the healthcare related data. PACS Cloud Gateway is used to access PACS Cloud archive, which provides a high security level and without the interference of cloud's provider.

\subsubsection{Data management in Health care institutions using Cloud Computing solutions}

Similar to clinical data archiving management and analyzing the stored data is also very important. One of the solutions proposed for this is by automating this process using sensors attached to the medical equipment's. Management and security is addressed by services architecture proposed in a paper [7].

\subsubsection{Health support system}

During the emergency management, the previous data of patients in that situation and pre-hospital care helps to provide proper medication and treatment, eliminating the risk of errors. The information collected describes a cloud-based service-oriented architecture (SOA) which implements the records of the details of emergency patients and provide the flexibility where the information is in a distributed and ubiquitous manner that supports several platforms and applications. In a study healthcare services are provided through a telemedicine oriented Emergency Health Support System (EHSS) is examined.

\subsubsection{Health support system}

Few mobile based cloud applications present in the medical field are:

- Comprehensive health monitoring services enable patients to be monitored at anytime and anywhere through broadband wireless communications.

- Health-aware mobile devices detect pulse-rate, blood pressure, and level of alcohol to alert healthcare emergency system.

- Intelligent emergency management system can manage and coordinate the fleet of emergency vehicles effectively and in time when receiving calls from accidents or incidents.

- Pervasive access to healthcare information allows patients or healthcare providers to access the current and past medical information.

- Pervasive lifestyle incentive management can be used to pay healthcare expenses and manage other related charges automatically. 
Table 1. Actual Healthcare needs and existing solution

\begin{tabular}{|c|c|}
\hline Needs & Existing Solution \\
\hline $\begin{array}{c}\text { Store large amount of data: } \\
\text { e.g. DICOM Images. }\end{array}$ & $\begin{array}{c}\text { PACS, Distributed Storage, } \\
\text { Storage on GRID. }\end{array}$ \\
\hline $\begin{array}{c}\text { Management of patient's } \\
\text { data: e.g. anamnesis, } \\
\text { patologies, sintoms, medical } \\
\text { records. }\end{array}$ & $\begin{array}{c}\text { Federated databases, specific } \\
\text { software for db interrogation. } \\
\text { EHR-EPR. }\end{array}$ \\
\hline $\begin{array}{c}\text { Needs for specific software } \\
\text { and hardware for data } \\
\text { processing. }\end{array}$ & $\begin{array}{c}\text { Distributed system, Private } \\
\text { server architecture. }\end{array}$ \\
\hline $\begin{array}{c}\text { Software and hardware } \\
\text { scalability for data } \\
\text { management. }\end{array}$ & Private architecture. \\
\hline $\begin{array}{c}\text { Needs to extend the } \\
\text { authorizations and to } \\
\text { warrant security policy. }\end{array}$ & Private architecture \\
\hline
\end{tabular}

\subsubsection{Issues in Mobile Computing}

As mobile devices has limitation to many issues, making use of cloud computing for the elimination of those issues. There are various disadvantages for implementing cloud in mobile devices.

- Having the limited resources in mobile devices it makes difficult to implement cloud. The resources might be computing power, limited battery and low quality display.

- Network related issues like Bandwidth, latency, availability and heterogeneity exists in mobile devices.

- The smart phones almost resembles the computers, thus facing many security related problems. Some of the security issues like device security, privacy of mobile user and securing data on cloud should be handled carefully. Also use of GPS can also prove to be a treat.

- Bandwidth is also a limitation in mobile device users.

- Service availability becomes the major concerns with Wi-Fi connection rather than wired connection. Few mobile based cloud applications present in the medical field are:

As discussed above the security plays the major concern in using cloud computing in mobile devices. The data files of mobile users are very sensitive as, there might be changes made to the file or it might be modified. Owner has to take care of the files created. Securing the mobile applications or application model is also important because these provide better services to mobile users by utilizing cloud resources. These mobile application models use the services of the cloud to increase the capability of a mobile device [8].

Interoperability is the current health IT buzzword because it's the essential ingredient in creating a system that benefits patients, doctors and hospitals. The system has to have the ability of two or more systems or components to exchange information and to use the information that has been exchanged. Mobile cloud computing is aiming in providing the same for the users.

But the never ending issue will always be with respect to security and privacy. The owner of the data should have control over it. Cloud should also provide authentication and not provide unlimited computational resource utilization for untrustworthy entities.

\section{SYSTEM ARCHITECTURE}

The architecture of the proposed model of a cloud computing based Secure Medical Data Management is presented in Figure 1. The architecture consists of a Cloud Service Provider, User Application, Data Security and User Authentication components. The Patient Records are encrypted by Data Security and User Authentication. These encrypted records are stored and managed in the Cloud Service Provider which is the server end. These records are retrieved from cloud storage when requested by a user and displayed on the mobile device after decryption.

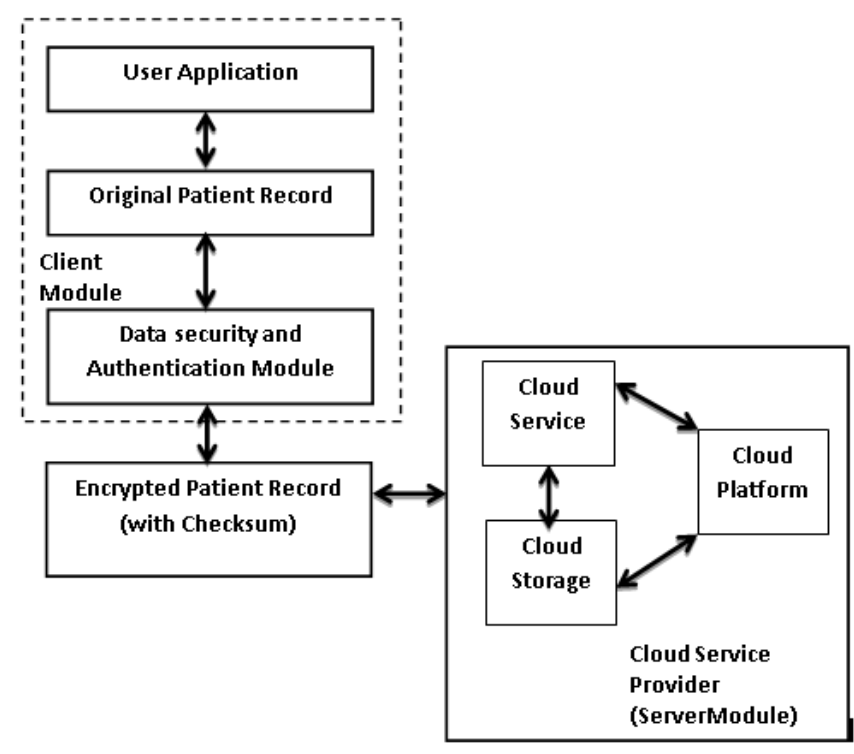

Fig 1: System architecture of secure medical data management

The User Application is designed in such a way that the health records and prescriptions of patients are made available to them on their smartphones. The application is designed to provide different access to the users based on their roles as: (1) Doctors can edit update and view the medical record, (2) Patients can only view the record, (3) Medical Data Management Administrator can create, delete, update records.

In the proposed model, component of Data Security and User Authentication protects medical records and prevents unauthorized access which is powered by the MD5 Hash Algorithm. This algorithm can be used for storing the passwords of each user which is used during user authentication. The Original Patient Record file can be appended with a checksum generated by MD5 which helps in confidentiality along with authentication. A Password-Based Encryption (PBE) is used to encrypt patient record which derives an encryption key from a password for each user. In order to make the task of getting from password to key very time-consuming for an attacker, implementation can mix in a random number, known as a salt, to create the key.

Any Cloud Service Provider (trail version) which provides resources for storage and service can be used for records storage and accessing them. The connection to the Cloud that allows record creation, modification, retrieval, updating and deletion can be done using internet services and REST API concepts like HTTP URLs. Instead of a service provider an open source toolkit can be used for the experiment setup. 
In this paper features like data storage, bandwidth consumption and network traffic, and failure recovery related to cloud platform are assumed to be correct and do not hinder the performance of application. It must also be assumed that, the user making request for a medical record has a smartphone with Internet connectivity up and running to connect to the Cloud. The scope of this work proposed is entitled as data security of the medical record and ease in accessing of data by the user

\section{CONCLUSION}

With so much attention paid to the data generated by electronic health instruments the provider organization need to make a special effort not to lose data, which might be helpful or provide a key opportunity for the infrastructure improvement. With the new infrastructure technology which will reduce the cost, improve performance and accelerate efforts to achieve healthy world. Organizations in this sector do not compromise with the quality of care or patient safety. Cloud computing is one promising technology those healthcare organizations can use to achieve these results.

Future research work could be implementation of a mobile healthcare information management system based on Cloud Computing and Android OS. However there are many issues that still needed the attention like security of Mobile Cloud Computing. Authentication and Confidentiality plays the major role with the healthcare data. To keep the data secure various authentication techniques should be used to check whether the user is trusted. It has to be aimed to implement the proposed work in a cloud based environment and provide strong security and privacy implementations to the medical data at cloud computing server side. Image operations will be provided at mobiles with the interface like zoom in / zoom out at the time of viewing and analyzing. Also healthcare standards for data maintenance and communication can be implemented.

\section{REFERENCES}

[1] Health Information Systems (HIS). PHIN [Online] http://phinnetwork.org/Resources/HIS.aspx

[2] World Health Organization, "Toolkit on monitoring health systems strengthening, Health Information Systems", June 2008.

[3] Cisco, "Cloud computing in healthcare".

[4] Mobile cloud computing. Wikipedia [Online] en.wikipedia.org/wiki/Mobile_cloud_computing

[5] Charalampos Doukas, "Mobile Healthcare Information Management utilizing Cloud Computing and Android OS" 32nd Annual International Conference of the IEEE EMBS, Buenos Aires, Argentina, August 31 - September 4, 2010.

[6] A new era for the healthcare industry. Cloud computing changes the game (references).

[7] Carmelo Pino and Roberto Di Salvo, "A Survey of Cloud Computing Architecture and Applications in Health" Proceedings of the 2nd International Conference on Computer Science and Electronics Engineering (ICCSEE 2013).

[8] Dhammapal Tayade, "Mobile Cloud Computing: Issues, Security,Advantages, Trends," in International Journal of Computer Science and Information Technologies, Vol. 5 (5) , 2014, 6635-6. 